\title{
Laparoscopic splenectomy for splenomegaly using a homemade retrieval BAG
}

\author{
Chia-Hung Su, Tzu-Chieh Yin, Che-Jen Huang, Wen-Chieh Fan, Jan-Sing Hsieh \\ Department of Surgery, Kaohsiung Medical University Hospital, Kaohsiung Medical University, Taiwan
}

Videosurgery Miniinv (e-pub, ahead of print) DOI: 10.5114/wiitm.2011.35433

\begin{abstract}
Introduction: Operating on an enlarged spleen via the laparoscopic approach presents several challenges. A homemade bag may facilitate retrieval of the enlarged spleen assisted by a laparoscope and save medical expense.

Aim: To assess the feasibility and safety of laparoscopic splenectomy for moderate or massive splenomegaly using our technique and a homemade retrieval bag.

Material and methods: Fifty patients underwent laparoscopic splenectomy for moderate or massive splenomegaly which was defined as the major axis exceeding $17 \mathrm{~cm}$ by abdominal computed tomography. A homemade retrieval bag made from a commercial sterile infusion container which costs about US\$ 1-2 per piece was used for spleen retrieval. Two transabdominal sutures for suspension of the retrieval bag were made to aid specimen removal in this technique.

Results: There were 31 males and 19 females with mean age of $56 \pm 11$ years. Laparoscopic splenectomy was successfully completed in 49 of these 50 patients. Overall, mean operative time was $149 \pm 31$ min (range: 100-252 min). Median estimated blood loss was $189 \pm 155 \mathrm{ml}$ (range: 50-920 ml). There were 12 minor complications but no mortality. Time to discharge after surgery ranged from 3 to 9 (mean: $4.7 \pm 1.7$ days). The average splenic weight was $729 \pm 74 \mathrm{~g}$ (range: 632-930 g).

Conclusions: Our preliminary results indicate that laparoscopic splenectomy is feasible and safe for moderate or massive splenomegaly and may be a well-tolerated alternative to open splenectomy. Not only is the cost of our homemade retrieval bag low, but also it is easy to make and ready to use.
\end{abstract}

Key words: laparoscopic splenectomy, splenomegaly, homemade retrieval bag.

\section{Introduction}

Laparoscopic splenectomy (LS) has been adopted as a feasible and safe procedure for management of various hematological disorders including idiopathic thrombocytopenic purpura, hematologic malignancy, and hereditary spherocytosis [1-7]. The laparoscopic approach offers several advantages over open splenectomy, such as shorter hospital stay, decreased blood loss, and low perioperative morbidity [1-7].
However, operating on an enlarged spleen via the laparoscopic approach presents several challenges including limited working space, difficulty with organ retraction and specimen retrieval, although massive splenomegaly is not an absolute contraindication [810]. In addition, splenomegaly may increase the risk of life-threatening hemorrhage, and the incidence of conversion to open splenectomy in such patients [1113]. Over the past decade, surgical techniques and instrumentation have been improved to overcome 
the difficulty of laparoscopic management for an enlarged spleen.

\section{Aim}

We have developed a modified method using our homemade bag, which may facilitate retrieval of the enlarged spleen assisted by a laparoscope. In this study, we intend to assess the feasibility and safety of LS, performed by our method, for treatment of moderate massive splenomegaly.

\section{Material and methods}

Between January 2005 and December 2010, 50 patients underwent LS for moderate massive splenomegaly in our department. Moderate massive splenomegaly is defined as a spleen weighing over $600 \mathrm{~g}$ or the major axis of the spleen exceeding $17 \mathrm{~cm}$ by abdominal computed tomography (CT). Preoperatively, all of the patients had detailed physical examination, chest radiograph, and routine biochemical and hematological tests. In addition, imaging investigations (ultrasonography, computed tomography, and magnetic resonance imaging) were conducted to determine the spleen size and vascularities. Data collection included patient demographics, diagnosis, operative details, and postoperative morbidity and mortality. Splenic weight was the aggregate weight of the morcellated and aspirated splenic tissue as measured in the pathology laboratory. At least one week prior to surgery, all patients received a meningococcal and pneumococcal vaccine. Antibiotic prophylaxis was administered preoperatively and continued postoperatively. Patients with hemoglobin

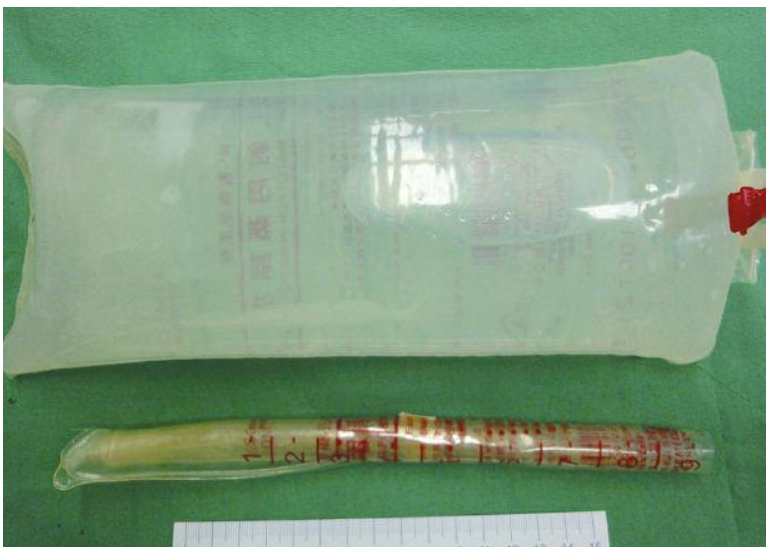

Figure 1. The rolled and unrolled versions of the commercial bag are shown
$<8 \mathrm{~g} / \mathrm{dl}$ or platelets $<50000 / \mathrm{mm}^{3}$ were administered fresh whole blood or platelet-rich plasma, respectively, and in most cases it proved unnecessary.

\section{Technique of laparoscopic splenectomy}

\section{Patient position}

The patient was positioned in a lateral decubitus position with the left side up and a flank cushion placed under the contralateral side, thus enlarging the space between the costal margin and the iliac crest. The left arm was extended and suspended. The surgeon faced the patient while the assistant stood at the right side of the operator.

\section{Trocar placement}

Initially, the peritoneal cavity is accessed via the open technique under direct vision and carbon dioxide is then inflated to create a pneumoperitoneum after insertion of a 12-mm trocar below the inferior tip of the spleen, parallel to the left costal margin. One 5-mm trocar is inserted under the xyphoid and another 12-mm trocar is placed midway between the $5-\mathrm{mm}$ and $12-\mathrm{mm}$ trocars for dissection and hilar devascularization. A third 12-mm trocar, used for the laparoscope, is inserted at the umbilicus.

\section{Surgical technique}

The surgical procedure is greatly facilitated by the LigaSure $^{\mathrm{TM}}$ (Valleylab ${ }^{\mathrm{TM}}$, Tyco Healthcare Group, Colorado, USA) vessel sealing system. The operation begins to divide the gastrosplenic ligament between the stomach and spleen to enter the lesser sac and to expose the tortuous pulsating splenic artery. After the splenic artery is intracorporeally ligated, the splenocolic ligament was divided to separate the colon. The splenorenal and retrosplenic ligaments are exposed and can then be divided, after mobilizing the splenophrenic ligament. Once the spleen is adequately mobilized and the tail of the pancreas is separated from the splenic hilum, an endoscopic stapler (Ethicon Endosurgery, Cincinnati, Ohio) is used to divide the splenic hilum and the short gastric vessels. Cautery is then used to divide the remaining attachments to the parietal peritoneum and diaphragm.

\section{Manufacture of the retrieval bag}

The homemade retrieval bag is made from a commercial sterile intravenous infusion container (Baxter 
Healthcare, USA), which is a foldable, flexible and waterproof PVC (polyvinyl chloride) bag (Figure 1). The price of this bag sold in this country is about one to two US dollars per piece. This bag has a capacity of $1000 \mathrm{ml}$ in volume, measuring $26 \mathrm{~cm} \times 15 \mathrm{~cm}$ in size. It is extremely tough and resistant to perforation while morcellating the spleen. The side with the infusion port of the container is then cut off to create a bag mouth with a short upper lip and a long lower lip. The storage capacity of the bag is large enough to contain a massive spleen up to $900 \mathrm{~g}$ in weight. A bigger bag of $2000 \mathrm{ml}$ in volume can also be adopted in this technique.

\section{Retrieval of the specimen}

After freeing the spleen, the retrieval bag is rolled into a cigar shape and inserted through the port site into the abdominal cavity (Figure $2 \mathrm{~A}$ ). The bag is deployed and laid in the left abdominal cavity, with the mouth orientated to the splenic fossa where the resected spleen is placed (Figure $2 \mathrm{~B}$ ). Two transabdominal suture sites are selected below the level of the lateral two port sites. A straight needle with suture is inserted into the abdominal cavity to penetrate the lateral side of the upper lip of the bag mouth (Figure $3 \mathrm{~A}$ ). The suture is then pulled through the abdominal wall by a specific hookpin [14] and tied on the abdominal skin. Another similar suture is made on the medial side of the upper lip. Finally, the whole upper lip of the bag mouth is thus anchored and fixed to the peritoneum of the anterior abdominal wall (Figure $3 \mathrm{~B}$ ). At this time, the laparoscope was changed to the middle one of the three subcostal trocars. By this way, the operative field would not be obscured by the large retrieval bag and the bag can easily be opened by holding and keeping the lower lip of the bag mouth tucked under the spleen with a 5-mm grasper (Figure $4 \mathrm{~A}$ ). Another grasper is inserted into the abdominal cavity to pull the spleen into the bag (Figure $4 \mathrm{~B}$ ). After the spleen is already placed in the bag, the two anchoring sutures are loosened and the edges of the bag are brought out of the abdomen via the lateral port. A ring forceps is used to morcellate the splenic tissue to facilitate extraction. A drainage tube is routinely placed in the splenic area in patients with portal hypertension or intraoperative blood loss $>200 \mathrm{ml}$. The tube was then removed if the daily amount of the discharge decreased to $50 \mathrm{ml}$ and the color became clear.

\section{Results}

Patient characteristics and indications for surgery are shown in Table I. Laparoscopic splenectomy was successfully completed in 49 of these patients. There was one conversion to an open procedure due to massive intraoperative bleeding. Overall, mean operative time was $149 \pm 31$ min (range: 100-252 min). Estimated blood loss was minimal in the majority of patients with only 3 patients experiencing blood loss
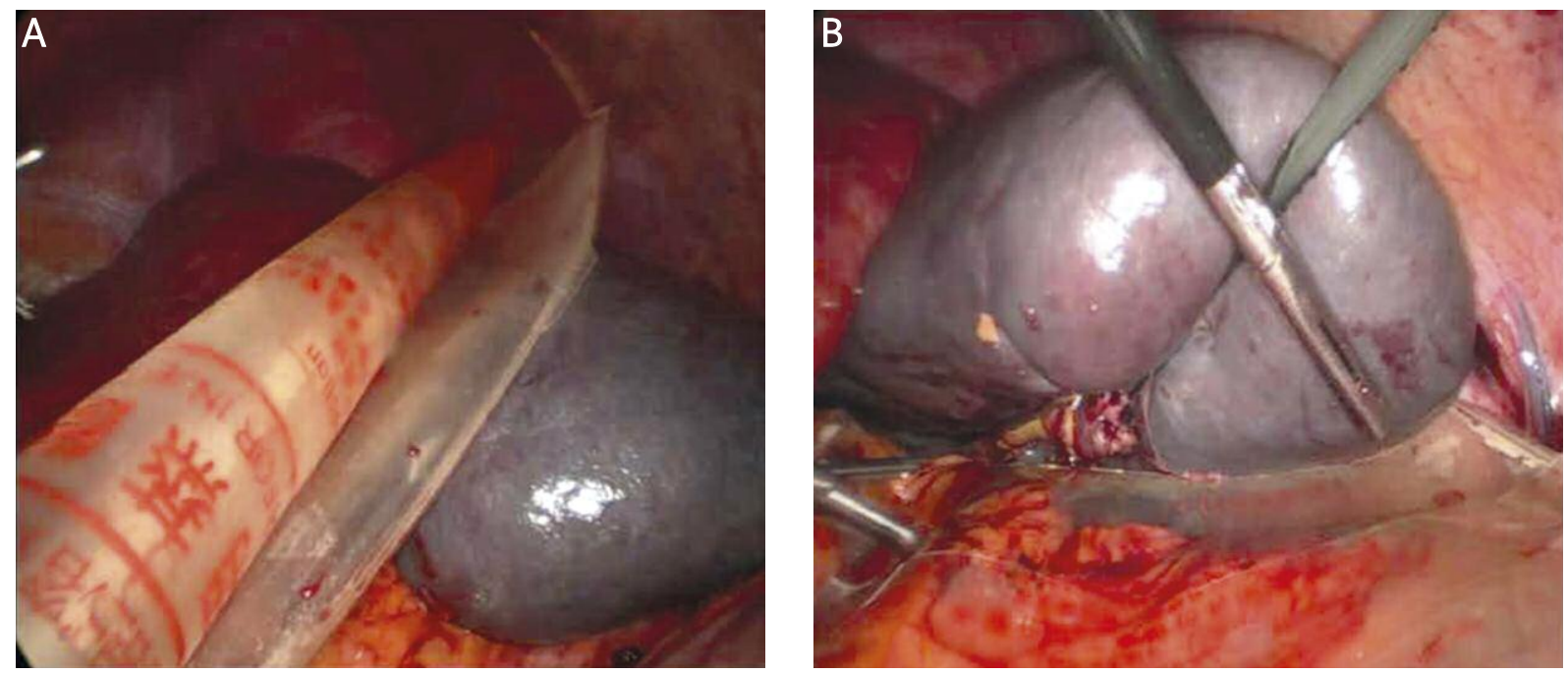

Figure 2. The rolled retrieval bag is inserted through the port site into the abdominal cavity (A). The bag is deployed and laid in the left abdominal cavity and the resected spleen is placed on the lower lip of the bag (B) 

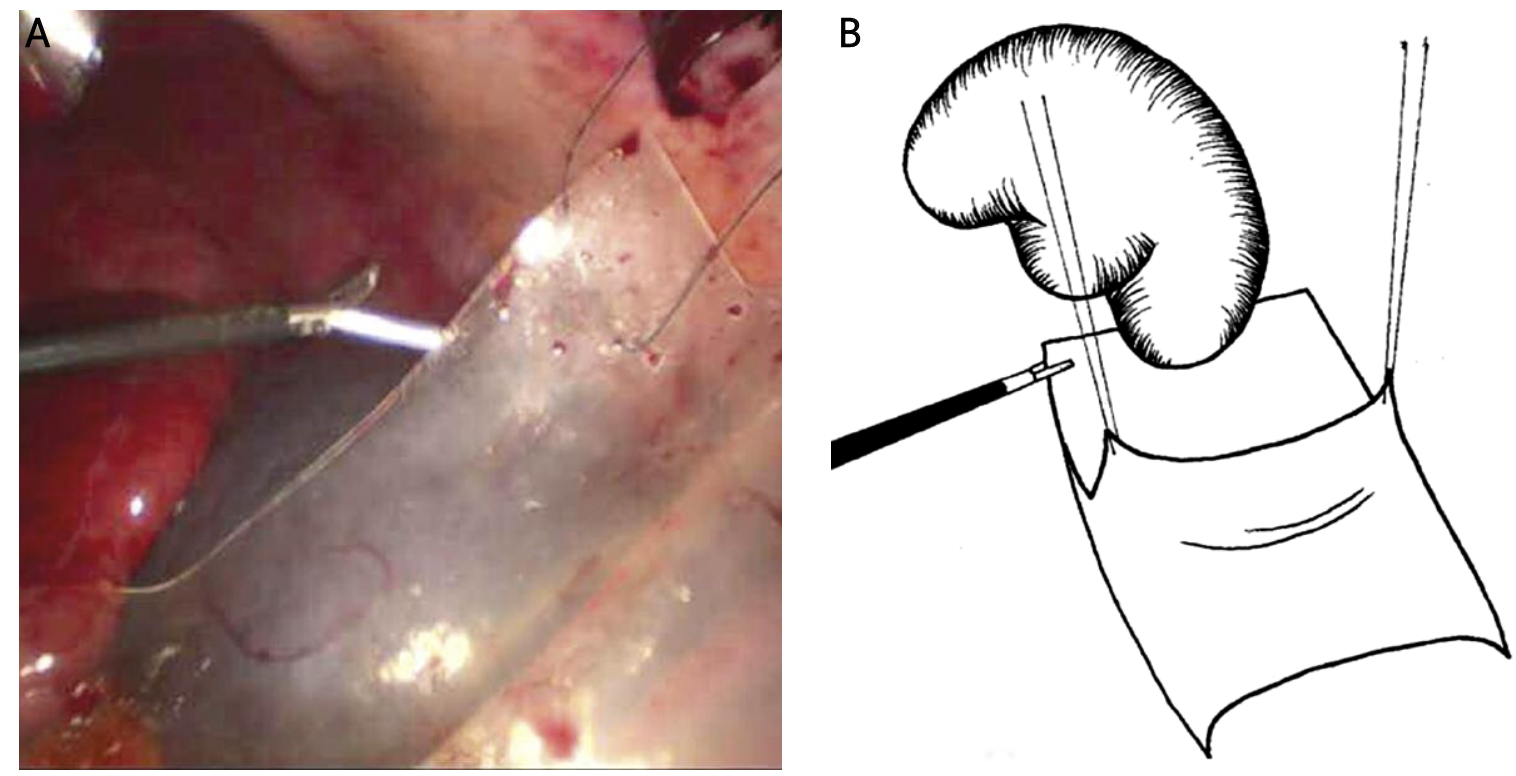

Figure 3. A transabdominal suture is made on the lateral side of the upper lip of the bag mouth (A). Another similar suture is made on the medial side of the upper lip. The bag can easily be opened by holding and keeping the lower lip of the bag mouth tucked under the spleen with a 5-mm grasper (B)
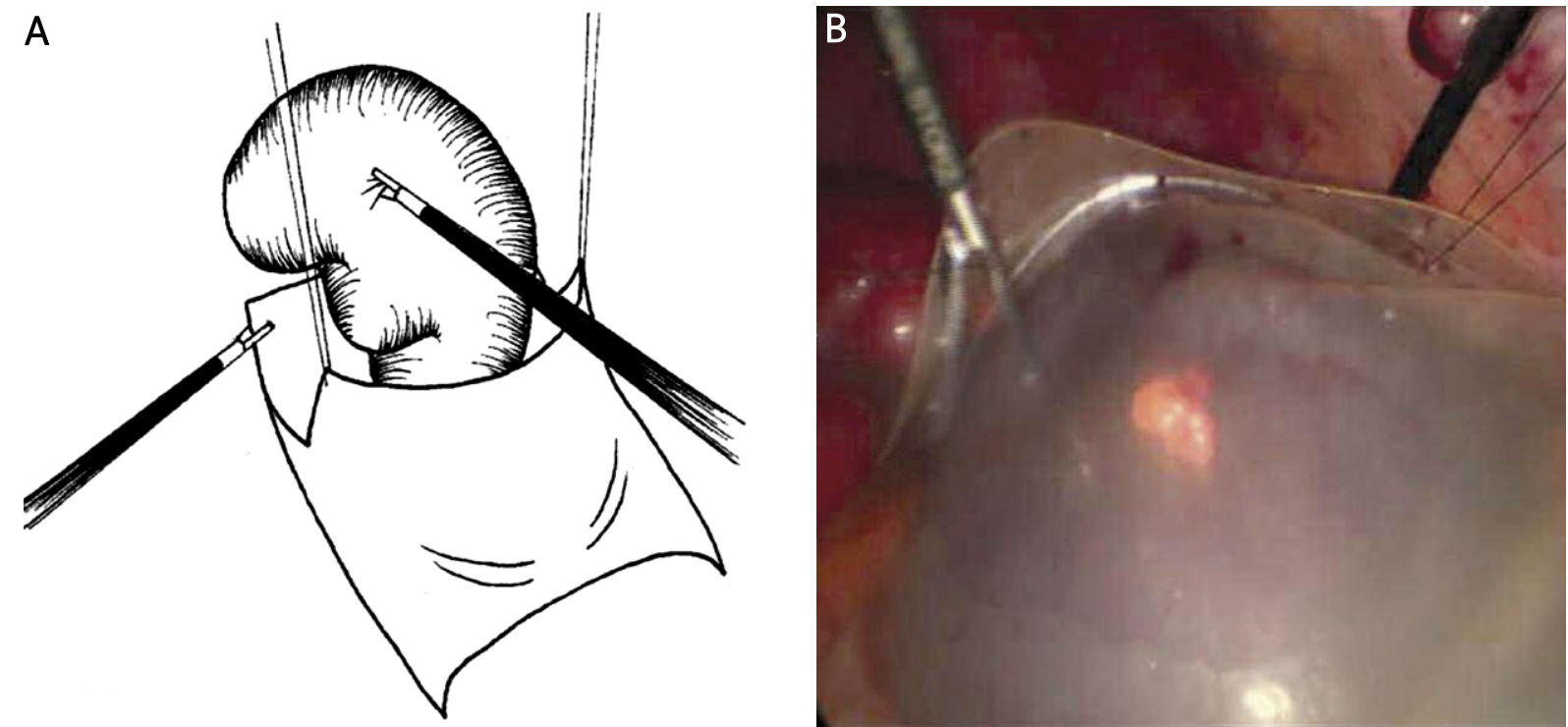

Figure 4. Another grasper is inserted into the abdominal cavity to pull the spleen into the bag, while the upper lip of the bag is suspended by two transabdominal sutures (A). The major portion of the spleen has been placed in the retrieval bag (B)

greater than $500 \mathrm{ml}$. Median estimated blood loss was $189 \pm 155$ cc (range: 50-920 cc). The drainage tube could be removed within 4 days postoperatively in the great majority of patients. There were no other major complications and no mortality. Out of the 50 patients with LS, 2 (4.0\%) presented with trocar site infection and 5 had postoperative mild fever without significant leukocytosis for a short period. Minor complications and the period of hospital are shown in Table II. In all patients the aggregated weights of morcellated splenic tissues were more than $600 \mathrm{~g}$ (mean: $729 \pm 74$ g; range: $632-930 \mathrm{~g}$ ). Time to discharge after surgery ranged from 3 to 9 (mean: $4.7 \pm 1.7$ days). 
Table I. Patient characteristics and diagnosis of the disorders

\begin{tabular}{|c|c|}
\hline Age [years] & 20-73 (mean, $56 \pm 11)$ \\
\hline Sex (male : female) & $31: 19$ \\
\hline Diagnosis & $n$ \\
\hline Liver cirrhosis & 40 \\
\hline Hereditary spherocytosis & 5 \\
\hline$\beta$-Thalassemia & 3 \\
\hline Splenic abscess & 1 \\
\hline Splenic cyst & 1 \\
\hline \multicolumn{2}{|c|}{ Child-Pugh classification for liver cirrhosis } \\
\hline A & 25 \\
\hline B & 13 \\
\hline C & 2 \\
\hline
\end{tabular}

\section{Discussion}

Successful LS with normal-sized and mildly enlarged spleens has prompted many surgeons to increase the use of this procedure in patients with massive splenomegaly $[4,8-10]$ since it was first described in 1991 [1]. In patients with severe liver disease, thrombocytopenia secondary to hypersplenism is a clinical feature that may represent an obstacle to chemotherapy, and anti-viral treatment. Previously patients presenting portal hypertension were not indicated for $L S$, most likely due to the risk of life-threatening hemorrhage and difficulties in achieving hemostasis in such patients $[15,16]$. Recently it has been reported that laparoscopic splenectomy in patients with hepatitis C cirrhosis can be done safely to allow application of antiviral treatment and potentially avoid transplantation [17]. Moreover, it has been reported that an enlarged spleen can be removed successfully by laparoscopy with improvement of the instrument and refinement of the techniques of $\mathrm{LS}$, although massive splenomegaly was once considered as a relative contraindication to LS, especially hypersplenism secondary to liver cirrhosis [8-10, 15, 16]. In this series, we also confirmed that LS is feasible and safe in cirrhotic patients with hypersplenism. Rarely, total or partial splenectomy via the laparoscopic approach may be employed as a surgical alternative for splenic infarction due to sleeve gastrectomy or sclerosing angiomatoid nodular transformation of the spleen $[18,19]$.
Table II. Perioperative data in patients with laparoscopic splenectomy $(n=50)$

\begin{tabular}{|lc|}
\hline Operative time $[\mathrm{min}]$ & $100-252($ mean $149 \pm 31)$ \\
\hline Blood loss [ml] & $50-920($ median $189 \pm 155)$ \\
\hline Postoperative complications & $n$ \\
\hline Trocar site infection & 2 \\
\hline Fever & 5 \\
\hline Flank hematoma & 5 \\
\hline Hospital stay [days] & $3-9($ mean 4.7 \pm 1.7$)$ \\
\hline Splenic weight [g] & $610-930($ mean $729 \pm 74)$ \\
\hline
\end{tabular}

A few potential difficulties have been encountered in the laparoscopic approach to massive splenomegaly. During LS for splenomegaly the operative field in the abdominal cavity is diminished by an enlarged spleen, and intra-abdominal manipulation of the bulky organ becomes more technically difficult. To solve the difficulty of retraction and prevent the risk of massive hemorrhage, we suggest early ligation of the splenic artery before mobilization of the spleen to shrink the size of the spleen by facilitating autotransfusion of the sinusoid blood. Laparoscopic splenectomy can be more secure to manipulate through this procedure. Several reports have indicated that early ligation of the splenic artery increases the safety of the surgery and reduces blood loss [17, 20]. The use of preoperative splenic embolization has been reported [21, 22] but does not appear necessary if the splenic blood supply is controlled early. Retrieval of the massively enlarged spleen is another obstacle to be overcome and may be the most challenging step of $L S$. There are several kinds of methods and various devices used for this purpose [23-25]. Usually a commercial endoscopic bag is employed for spleen removal. However, it can be very difficult, and even impossible, to introduce the enlarged organ into a small endoscopic bag. It has been found that the process of deploying and opening the bag for specimen retrieval, using conventional laparoscopic instruments, may be annoying and time-consuming [23-25]. Sometimes, a separate abdominal incision $[26]$ or the hand port technique $[23,27]$ is used when attempting to remove massive spleens from the abdomen laparoscopically. However, extending the wound of the hand port site or making another large secondary abdominal incision defeats the advan- 
tages associated with laparoscopic surgery. It may not only add operative time but also increase the expense of laparoscopic surgery. Poulin and Thibault [4] proposed the intraperitoneal morcellation of the spleen and its retrieval in several pieces, followed by intraperitoneal irrigation to avoid splenic tissue implantation. However, the risks of subsequent splenosis due to splenic tissue deposition and injury of surrounding structures during morcellation should be avoided.

In this study two patients (4\%) developed surgical site infection which could be controlled after intensive and adequate wound care. It is well known that potentially life-threatening infection is a major longterm risk in post-splenectomized or hyposplenic patients. Early and local surgical site infection also occurs much more often than could be expected [28]. However, the benefits of local or systemic antibiotic prophylaxis against local infection remain questionable [28]. Contrarily, it is generally accepted to prevent overwhelming post-splenectomy infection (OPSI) by appropriate vaccination and life-long antibiotic chemoprophylaxis [29]. There is a consensus among the various guidelines regarding immunization prior to elective splenectomy and post-trauma. Asplenic individuals should be educated and repeatedly reminded about the risks and health precautions which are required to avoid infectious hazards [30]. Regarding the necessity of routine drainage after laparoscopic splenectomy, several studies confirmed that the length of hospital stay is shorter when drainage is abandoned [31]. Moreover, "drain fever syndrome", which is a febrile symptom without accompanying signs of infection, may disappear and patients with postoperative abdominal pain also noticed considerable improvement after the drain's removal [31].

We have described a simple technique, using a self-designed retrieval bag, to facilitate the removal of massively enlarged spleens laparoscopically. The ideal bag for specimen retrieval should be easy to use and keep wide open during the entrapping procedure. It must also be strong enough to allow for the safe morcellation of the spleen and to prevent causing damage to the surrounding organs and spillage of the splenic tissue in the peritoneal cavity. Our technique facilitates removal of the massively enlarged spleen from the abdomen, and can be accomplished with low morbidity and low risk of splenosis. No additional incision of the abdominal wall or intraperi- toneal fragmentation of the spleen is required. Compared to other techniques, our proposed method provides distinct advantages over other methods of controlling the retrieval bag. The bag is also readily available and inexpensive and the technique is not difficult. This technique significantly shortens this phase of spleen retrieval and thus the operative time. Our preliminary experiences are consistent with the conclusion that laparoscopic-assisted surgery for massive spleens is both feasible and effective, and may be a well-tolerated alternative to open splenectomy.

\section{References}

1. Delaitre B, Maignien B. Laparoscopic splenectomy: one case. Presse Med 1991; 44: 2263.

2. Carroll BJ, Phillips EH, Semel CJ, et al. Laparoscopic splenectomy. Surg Endosc 1992; 6: 183-5.

3. Kathkouda N, Hurtwitz MB, Rivera RT, et al. Laparoscopic splenectomy. Outcome and efficacy in 103 consecutive cases. Ann Surg 1998; 228: 568-78.

4. Poulin EC, Thibault C. Laparoscopic splenectomy for massive splenomegaly: operative technique and case report. Can I Surg 1995; 38: 69-72.

5. Park A, Marcaccio M, Sternbach M, et al. Laparoscopic versus open splenectomy. Arch Surg 1999; 134: 1263-9.

6. Rege RV, Joehl RJ. A learning curve for laparoscopic splenectomy at an academic institution. J Surg Res 1999; 81: 27-32.

7. Trías M, Targarona EM, Espert JJ, et al. Impact of hematological diagnosis on short and long term follow up after laparoscopic splenectomy. Surg Endosc 2000; 14: 556-60.

8. Yee JC, Akpata MO. Laparoscopic splenectomy for congenital spherocytosis with splenomegaly: a case report. Can J Surg 1995; 38: 73-6.

9. Terrosu G, Donini A, Baccarani U, et al. Laparoscopic versus open splenectomy in the management of splenomegaly: our preliminary experience. Surgery 1998; 24: 839-43.

10. Targarona EM, Espert JJ, Balague C, et al. Splenomegaly should not be considered a contraindication for laparoscopic splenectomy. Ann Surg 1998; 228: 35-9.

11. Grahn SW, Alvarez J 3rd, Kirkwood K. Trends in laparoscopic splenectomy for massive splenomegaly. Arch Surg 2006; 141: 755-62.

12. Patel AG, Parker JE, Wallwork B. Massive splenomegaly is associated with significant morbidity after laparoscopic splenectomy. Ann Surg 2003; 238: 235-40.

13. Targarona EM, Espert JJ, Cerdan G, et al. Effect of spleen size on splenectomy outcome. Surg Endosc 1999; 13: 559-62.

14. Hsieh JS, Wu CF, Chen FM, et al. Simple laparoscopic gastrostomy using a homemade hook-pin. Endoscopy 2007; 39 Suppl 1: E226.

15. Hashizume M, Tanoue K, Morita M, et al. Laparoscopic gastric devascularization and splenectomy for sclerotherapy- resistant esophagogastric varices with hypersplenism. J Am Coll Surg 1998; 187: 263-70. 
16. Kercher KW, Carbonell AM, Heniford BT, et al. Laparoscopic splenectomy reverses thrombocytopenia in patients with hepatitis C cirrhosis and portal hypertension. J Gastrointest Surg 2004; 8: 120-6.

17. Nicholson IA, Falk GL, Mulligan SC. Laparoscopically assisted massive splenomegaly: a preliminary report of the technique of early hilar devascularization. Surg Endosc 1998; 12: 73-5.

18. Budzyński A, Demczuk S, Kumiega B, et al. Sclerosing angiomatoid nodular transformation of the spleen treated by laparoscopic partial splenectomy. Videosurgery Miniinv 2011; 6: 249-55.

19. Michalik M, Budziński R, Orlowski M, et al. Splenic infarction as a complication of laparoscopic sleeve gastrectomy. Videosurgery Miniinv 2011; 6: 92-8.

20. Palanivelu C, Jani K, Malladi V, et al. Early ligation of the splenic artery in the leaning spleen approach to laparoscopic splenectomy. J Laparoendosc Adv Surg Tech A 2006; 16: 339-44.

21. Iwase K, Higaki J, Yoon HE. Splenic artery embolization using contour emboli before laparoscopic or laparoscopically assisted splenectomy. Surg Laparosc Endosc Percutan Tech 2002; 12: 331-6.

22. Poulin EC, Mamazza J, Schlachta CM. Splenic artery embolization before laparoscopic splenectomy. An update. Surg Endosc 1998; 12: 870-5.

23. Targarona EM, Balague C, Cerdan G, et al. Hand-assisted laparoscopic splenectomy (HALS) in cases of splenomegaly: a comparison analysis with conventional laparoscopic splenectomy. Surg Endosc 2002; 16: 426-30.

24. Zacharoulis D, O'Boyle C, Royston CM, et al. Splenic retrieval after laparoscopic splenectomy: a new bag. I Laparoendosc Adv Surg Tech A 2006; 16: 128-32.

25. Greene AK, Hodin RA. Laparoscopic splenectomy for massive splenomegaly using a Lahey bag. Am I Surg 2001; 181: 543-6.

26. Choy C, Cacchione R, Moon V, et al. Experience with seven cases of massive splenomegaly. I Laparoendosc Adv Surg Tech A 2004 14: 197-200.

27. Demetrius EM, Litwin MD, Darzi A. Hand-assisted laparoscopic surgery (HALS) with the handport system: initial experience with 68 patients. Ann Surg 2000; 231: 715-23.

28. Migaczewski M, Zub-Pokrowiecka A, Budzyński P, et al. Prevention of early infective complications after laparoscopic splenectomy with the Garamycin sponge. Videosurgery Miniinv 2012; 7: 105-10.

29. Brigden ML, Pattullo AL. Prevention and management of overwhelming postsplenectomy infection - an update. Crit Care Med 1999; 27: 836-42.

30. El-Alfy MS, El-Sayed MH. Overwhelming postsplenectomy infection: is quality of patients knowledge enough for prevention? Haematol J 2004; 5: 77-80.

31. Major P, Matłok M, Pędziwiatr M, et al. Do we really need routine drainage after laparoscopic adrenalectomy and splenectomy? Videosurgery Miniinv 2012; 7: 33-9. 